Review

\title{
Potential Role of Thymosin Beta 4 in Liver Fibrosis
}

\author{
Jieun Kim ${ }^{1}$ and Youngmi Jung ${ }^{1,2, *}$
}

1 Department of Integrated Biological Sciences, Pusan National University, 63-2 Pusandaehak-ro, Kumjeong-gu, Pusan 609-735, Korea; E-Mail: jieun@pusan.ac.kr

2 Department of Biological Sciences, Pusan National University, 63-2 Pusandaehak-ro, Kumjeong-gu, Pusan 609-735, Korea

* Author to whom correspondence should be addressed; E-Mail: y.jung@pusan.ac.kr; Tel.: +82-51-510-2262; Fax: +82-51-581-2962.

Academic Editor: Johannes Haybaeck

Received: 15 April 2015 / Accepted: 4 May 2015 / Published: 8 May 2015

\begin{abstract}
Liver fibrosis, the main characteristic of chronic liver diseases, is strongly associated with the activation of hepatic stellate cells (HSCs), which are responsible for extracellular matrix production. As such, investigating the effective regulators controlling HSC activation provides important clues for developing therapeutics to inhibit liver fibrosis. Thymosin beta 4 (T $\beta 4)$, a major actin-sequestering protein, is known to be involved in various cellular responses. A growing body of evidence suggests that $\mathrm{T} \beta 4$ has a potential role in the pathogenesis of liver fibrosis and that it is especially associated with the activation of HSCs. However, it remains unclear whether $\mathrm{T} \beta 4$ promotes or suppresses the activation of HSCs. Herein, we review the potential role of T $\beta 4$ in liver fibrosis by describing the effects of exogenous and endogenous $\mathrm{T} \beta 4$, and we discuss the possible signaling pathway regulated by $\mathrm{T} \beta 4$. Exogenous $\mathrm{T} \beta 4$ reduces liver fibrosis by inhibiting the proliferation and migration of HSCs. T $\beta 4$ is expressed endogenously in the activated HSCs, but this endogenous T $\beta 4$ displays opposite effects in HSC activation, either as an activator or an inhibitor. Although the role of T $\beta 4$ has not been established, it is apparent that $\mathrm{T} \beta 4$ influences HSC activation, suggesting that $\mathrm{T} \beta 4$ is a potential therapeutic target for treating liver diseases.
\end{abstract}

Keywords: thymosin beta 4; liver fibrosis; hepatic stellate cells 


\section{Introduction}

Liver disease, including liver cancer, is a major cause of morbidity and mortality worldwide [1-4]. The main cause of liver disease is viral, autoimmune, drug/toxin, alcohol and nonalcoholic fatty liver disease [5]. Liver fibrosis is the main hallmark of most chronic liver diseases and can progress into end-stage cirrhosis showing the altered hepatic function and the dysregulated regenerative process with formation of fibrous bands [6]. Liver fibrosis typically occurs in response to hepatic injury; however, progressive and/or severe damage leads to the massive deposition of extracellular matrix (ECM) proteins, promoting a distorted architecture of the liver [7]. These fibrous matrix proteins are mainly produced by activated hepatic stellate cells (HSCs), which are transdifferentiated from quiescent HSCs. The activated HSCs, which look like a myofibroblast, with long processes without lipid droplets, contribute to liver fibrosis by accumulating ECMs [8]. However, the underlying mechanisms of HSC activation are poorly understood, and no effective treatments have been found.

Thymosin beta 4 (T $\beta 4)$ is a highly conserved G-actin sequestering protein that exerts a broad range of functions, such as the promotion of cell migration, angiogenesis, and ECM synthesis [9]. Recently, aberrant $\mathrm{T} \beta 4$ has been shown to play a key role in the development of organ fibrosis [10]. In addition, it has been reported that $\mathrm{T} \beta 4$ is expressed in activated HSCs during liver fibrosis and that it influences the activation of HSCs [11,12]. These findings suggest that $\mathrm{T} \beta 4$ is involved in the pathogenesis of liver fibrosis. Therefore, we review the functions and the related pathway of T $\beta 4$ in several types of tissues and/or cells and describe the endogenous and exogenous actions of T $\beta 4$ in liver to suggest how T $\beta 4$ is potentially involved in the pathogenesis of liver fibrosis.

\section{Thymosin Beta 4}

Thymosin isolated from the thymus gland was first described in 1966 by Goldstein and White [13]. The $\beta$-thymosin family is composed of biochemically and functionally distinct polypeptides. T $\beta 4$, $\mathrm{T} \beta 10$ and $\mathrm{T} \beta 15$, among the known family members, are found in humans [9]. The most abundant peptide in this family, T $\beta 4$, is a highly conserved and water-soluble, acidic polypeptide consisting of 43 amino acids and a molecular weight of $4964 \mathrm{Da}$ [14]. T $\beta 4$ is selectively crosslinked by factor XIIIa to various molecules, including actin, collagen, and fibrin [9]. It also acts as a major actin-sequestering molecule in all eukaryotic cells and is a potent regulator of actin polymerization in mammals [15]. Emerging evidence suggests that $\mathrm{T} \beta 4$ is involved in a number of cellular responses, such as angiogenesis, wound healing, hair growth, apoptosis, and inflammation [16-19]. T $\beta 4$, released by platelets in the region of injured dermis, can accelerate dermal wound healing by promoting cell migration, accelerating collagen deposition, and inhibiting both inflammation and apoptosis [20].

T $\beta 4$ promotes the survival of cardiac myocytes after ischemia, an effect that is mediated by the increased expression of vascular endothelial growth factor (VEGF) and activation of integrin-linked kinase (ILK) [21]. Recent studies have shown that T $\beta 4$ is overexpressed in malignant tumors, and it has been suggested that it is associated with metastatic capability and angiogenesis [16,22-25]. Induced expression of $\mathrm{T} \beta 4$ has been shown to enhance tumor growth and metastasis in melanoma cell lines and mouse fibrosarcoma [26]. In addition, T $\beta 4$-overexpressing human colon cancer cells have exhibited increased growth and invasion in transplanted mice with those cancer cells [25]. Huang et al. [27] revealed 
that $\mathrm{T} \beta 4$ overexpression promoted cell invasion and migration through the ILK/AKT/ $\beta$-catenin signaling pathway and triggered epithelial-to-mesenchymal transition (EMT) in colorectal carcinoma. $\mathrm{T} \beta 4$ also acts as a hypoxia-responsive regulator that controls cancer cell migration in angiogenesis and tumor metastasis [28].

These various cellular responses are regulated by the T $\beta 4$-mediated expression of several genes, such as specific proteases, laminin-5, and several inflammatory cytokines and chemokines. T $\beta 4$ increased the expression of laminin- 5 and transforming growth factor- $\beta$ (TGF- $\beta$ ) in cultured corneal cells, which in turn upregulated laminin-5- and TGF- $\beta$-induced cell migration and collagen synthesis, respectively [29]. T $\beta 4$ has been shown to influence the Wnt signaling pathways by regulating the activation of glycogen synthase kinase-3 (GSK-3) in the migration of gastric cancer cells [30]. Hepatocyte growth factor (HGF) promotes the upregulation of T $\beta 4$, which influences wound healing in human umbilical vein endothelial cells [31]. Thus, because T $\beta 4$ exerts a broad spectrum of functions by interacting with various molecules, it is important to research how $\mathrm{T} \beta 4$ is regulated and what its targets are, in order to understand the action mechanism of $\mathrm{T} \beta 4$.

\section{Liver Fibrosis and Hepatic Stellate Cells}

Liver fibrosis is a common wound-healing response to liver injury [32]. However, persistent and/or severe damage, such as continuous alcohol consumption, viral infection, toxins, and high-fat diets, impair the repair response, leading to the development of fibrotic scar and chronic wounds characterized by excessive inflammation and collagen deposition and the dysregulated proliferation of several types of hepatic cells [33]. The major type of cells contributing to fibrosis is HSC [7]. In the liver, HSCs reside in the space of Disse between hepatocytes and sinusoidal endothelial cells, and they extend their dendritic processes along the walls of the sinusoids. HSCs undergo transdifferentiation from "quiescent" HSCs into "activated/myofibroblastic" HSCs during hepatic injury [32]. In the quiescent stage, HSCs store perinuclear retinoid (vitamin A) droplets and synthesize glial fibrillary acidic protein (GFAP) [34]. As they are activated, retinoid droplets and GFAP are gradually lost, followed by development into myofibroblast-like cells with increased synthesis of ECM proteins and $\alpha$-smooth muscle actin ( $\alpha$-SMA) [33]. These activated HSCs migrate and proliferate effectively in response to a variety of cytokines and growth factors stimulated by liver injury [35]. The transdifferentiation, proliferation, and ECM production of HSCs are the important events that occur during liver fibrosis. Therefore, it is believed that developing effective strategies to suppress these events will reduce fibrosis or prevent a progression into chronic liver injury. In particular, many studies have been conducted on the transdifferentiation of quiescent HSCs into activated HSCs, as this process has been considered to be a possible therapeutic target to inhibit liver fibrosis [36,37].

Several factors, such as TGF- $\beta$, platelet-derived growth factor (PDGF), phosphatidylinositol 3-kinase $(\mathrm{PI} 3 \mathrm{~K})$ /protein kinase B (AKT), and hedgehog $(\mathrm{Hh})$, which have been reported to regulate hepatic fibrogenesis, are highly expressed in the damaged liver and stimulate the transition of quiescent HSCs and/or promote the proliferation of newly generated HSCs [35,38-40]. TGF- $\beta$, a well-known fibrosis-stimulating factor, plays a critical role in regulating HSC activation and ECM production in HSCs [41]. The PI3K/AKT pathway is strongly activated by the well-known mitogen PDGF and is required for the survival and proliferation of HSCs $[38,39,42]$. Hh signaling regulates the proliferation 
and activation of HSCs, leading to liver fibrosis $[43,44]$. In addition, TGF- $\beta$ and Hh have been known to promote epithelial-to-mesenchymal transition (EMT), which is an important pathogenic pathway that contributes to the excessive accumulation of activated HSCs, leading to collagen deposition during liver fibrosis $[44,45]$.

\section{Thymosin Beta 4 and Liver Fibrosis}

Although T $\beta 4$ is known to be expressed in the liver, the types of hepatic cells that specifically express T $\beta 4$ have not been well established. Based on the immunochemical data, Nemolato et al. [46] reported that hepatocytes expressed $\mathrm{T} \beta 4$ in the healthy human liver, whereas Paulussen et al. [47] reported that Kupffer cells (hepatic macrophages) express $\mathrm{T} \beta 4$ in the damaged liver. In addition, increased expression of $\mathrm{T} \beta 4$ has been reported in human hepatocellular carcinoma [48] and hepatoblastoma [49]. Recent studies with more evidence have revealed that activated HSCs express T $\beta 4$ in chronically damaged livers [11,12]. Because the activated HSCs are the main source of collagen production, investigation into the potential role of T $\beta 4$ in the activation of HSCs in the liver is needed. However, conflicting results in the regulation of T $\beta 4$ for HSC activation, inhibition, or promotion have been reported. Therefore, it is necessary to first examine the types of cells that express $\mathrm{T} \beta 4$ in order to identify the exogenous and endogenous roles of $\mathrm{T} \beta 4$ in the liver.

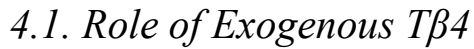

Several studies have suggested that $\mathrm{T} \beta 4$ treatment has an antifibrotic effect in the liver. Barnaeva et al. [50] demonstrated that T $\beta 4$-treated HSCs showed an upregulation of HGF and a downregulation of the PDGF- $\beta$ receptor at the RNA levels. The researchers have suggested that exogenous T $\beta 4$ has an antifibrogenic function in human HSCs because the level of HGF expression is reduced in fully activated HSCs [51] and PDGF- $\beta$ receptor is required for the activation of HSCs [52]. However, $\alpha$-SMA, a well-known marker of activated HSCs [53], and $\beta$-catenin and GSK-3 $\beta$, members of the Wnt pathway involved in the transdifferentiation of HSCs [54,55], were upregulated in a T $\beta 4$-dose-dependent manner. In addition, the expression of other ECM components, such as collagen $1 \alpha 2$, tissue inhibitor of metalloproteinase-1, and matrix metalloproteinase (MMP)-2, was not changed by T $\beta 4$. Although these results suggest that downregulation of the PDGF- $\beta$ receptor by T $\beta 4$ prevents the proliferation of HSC-derived myofibroblasts, there has been no explanation for the increased expression of other ECM members, especially $\alpha$-SMA, by T $\beta 4$. Further studies are required to prove the disparity in the actions of T $\beta 4$ in PDGF- $\beta$ receptor and $\alpha$-SMA, as both have been considered hallmarks of HSC differentiation into myofibroblasts. In 2011, Reyes-Gordillo et al. [56] reported that $\mathrm{T} \beta 4$ treatment prevented PDGF- $\beta \beta$-dependent proliferation and migration of cultured human HSCs by inhibiting PDGF- $\beta \beta$-dependent phosphorylation of AKT. In these studies, the researchers demonstrated that $\mathrm{T} \beta 4$ interrupted the movement of AKT into PI3K, blocking the phosphorylation of AKT by PI3K in HSCs treated with PDGF- $\beta \beta$ [56]. While exogenous T $\beta 4$ treatment successfully reduced the proliferation and migration of HSCs stimulated by PDGF- $\beta \beta$, when the activated HSCs were treated with $\mathrm{T} \beta 4$, these cells also showed decreased or unchanged expression of the genes under examination. Hence, it is possible that endogenous $\mathrm{T} \beta 4$ is combined with exogenous $\mathrm{T} \beta 4$ treatment, and that too high of a concentration of this combined T $\beta 4$ desensitizes the T $\beta 4$ 
signaling pathway, eventually blocking the proliferation of HSCs. In these two studies, it is noted that Barnaeva and Reyes-Gordillo did not examine the endogenous expression and role of T $\beta 4$ in HSCs. Nevertheless, Reyes-Gordillo et al. [57] demonstrated in 2012 that exogenous T $\beta 4$ treatment ameliorated acute liver damage induced by a single injection of carbon tetrachloride $\left(\mathrm{CCl}_{4}\right)$ in rats. The liver morphology is improved in the $\mathrm{CCl}_{4}+\mathrm{T} \beta 4$-treated group compared to the $\mathrm{CCl}_{4}$-treated group, assessed by hematoxylin and eosin staining. The hepatic protective effect of exogenous $\mathrm{T} \beta 4$ is mediated by inhibiting the upregulation of fibrotic markers (PDGF- $\beta$ receptor, $\alpha$-SMA, collagen $1 \alpha 2$ and fibronectin) and methyl-CpG-binding protein 2 (MeCP2) and the downregulation of adipogenic transcription factor peroxisome proliferator-activated receptor (PPAR $\gamma)$ at the RNA level. PPAR $\gamma$ expressed by quiescent HSCs is reduced by MeCP2 in activated HSCs [58]. The researchers suggested that T $\beta 4$ maintained the expression level of PPAR $\gamma$ by reducing MeCP2 expression, thus contributing to maintaining the quiescent phenotypic state of HSCs in rat livers [57]. However, the repair response, including activation of HSCs and inflammation, is a necessary process in the damaged liver [7]. The liver's health is dictated by a balance between the factors that regulate injury and repair. Liver injury stimulates a repair response, and when the repair response is inadequate, tissue damage persists and can progress. Therefore, defective repair responses permit mild liver injury to progress to cirrhosis [33]. Liver damage caused by a single $\mathrm{CCl}_{4}$ injection stimulated a repair response, and $\mathrm{T} \beta 4$ treatment clearly showed the improved morphology of a liver treated with $\mathrm{CCl}_{4}$, indicating that $\mathrm{T} \beta 4$ promotes liver regeneration. Therefore, the mechanism by which the repair process occurs without the activation of HSCs should be identified.

Taken together, exogenous $\mathrm{T} \beta 4$ has a role in attenuating liver fibrosis, a process that seems to be mediated by preventing the proliferation and migration of HSCs [50,56,57]. However, it is first necessary to investigate the types of cells that express endogenous $\mathrm{T} \beta 4$ in the liver, as well as whether endogenous T $\beta 4$ is actually expressed in activated or inactivated HSCs, in order to understand the role and mechanism underlying the effects of $\mathrm{T} \beta 4$ in the liver.

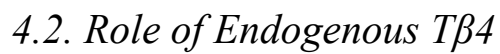

As the expression and function of T $\beta 4$ have been investigated recently in the liver, research on the types of cells that express T $\beta 4$ has attracted attention. Nemolato et al. [46] and Paulussen et al. [47] reported the expression of T $\beta 4$ in hepatocytes and Kupffer cells (CD 11b- or CD 68-positive cells), respectively. T $\beta 4$ expression these cells was assessed by single immunostaining for T $\beta 4$. T $\beta 4$ was suggested to indirectly control the migration of Kupffer cells, based on its ability to regulate the actin polymerization. [47]. Henkel et al. [59] also showed that Kupffer cells express T $\beta 4$ in liver sections of patients with hepatitis $\mathrm{C}$ virus (HCV), as examined by immunostaining. They suggested $\mathrm{T} \beta 4$ as a new potential biomarker for inflammatory hepatic lesions, although $\mathrm{T} \beta 4$ at the RNA level was not related with the degree of fibrosis [59]. However, they presented the T $\beta 4$-positive cells in HCV-infected liver only, although they examined the RNA level of T $\beta 4$ in several kinds of liver diseases. In addition, the effect of T $\beta 4$ expression in Kupffer cells was not investigated. Recently, Xiao et al. [11] and Kim et al. [12] provide direct evidence that activated HSCs express T $\beta 4$ in vivo and in vitro. However, Xiao and Kim suggested directly opposite interpretations of the role of T $\beta 4$ in the liver. Xiao et al. [11] demonstrated that LX-2 cells, the activated human HSC line, strongly expressed T $\beta 4$, and that depletion of T $\beta 4$ 
promoted the proliferation and migration of LX-2 cells via the activation of the PI3K/AKT signaling pathway. AKT (Ser473 and Thr308) and PI3K signaling components (p-85 regulatory subunit, PDK1 and GSK-3 $\beta$ ) were phosphorylated in the T $\beta 4$-suppressed LX-2 cells by small interfering RNA (siRNA) [11]. They also showed that $\mathrm{T} \beta 4$ was downregulated in both livers of bile-duct-ligated (BDL) rats and patient serum with cirrhosis. Bile duct ligation is a well-established model, in which the accumulated myofibroblasts or the activated HSCs produce excessive ECMs, leading to fibrosis [60]. Therefore, the livers of those rats should have displayed increased T $\beta 4$ expression, because the activated HSCs proliferate abundantly in those livers [61-63] and Xiao et al. reported an upregulation of T $\beta 4$ in these cells [11]. However, T $\beta 4$ levels decreased in the BDL livers, although it was difficult to compare T $\beta 4$ expression in a BDL liver with that of a healthy liver without evidence of T $\beta 4$ expression in the livers of control rats. The researchers also suggested that T $\beta 4$ depletion promoted HSC activation by exhibiting increased protein levels of vimentin and $\alpha$-SMA protein. If T $\beta 4$ inhibited HSC activation, higher levels of T $\beta 4$ should have been expressed in the inactivated HSCs, and HSCs should have been activated in the absence or lower expression of $\mathrm{T} \beta 4$. However, there was no explanation for how the activated HSCs expressed T $\beta 4$.

Kim et al. [12] recently suggested that activated HSCs express T $\beta 4$, which regulates the activation of HSCs. In their studies, T $\beta 4$ expression was upregulated in the fully activated LX-2 cells, the activated primary mouse $\mathrm{HSC}$, the fibrotic livers of human patients, and the $\mathrm{CCl}_{4}$-induced fibrotic livers of mice. The suppression of T $\beta 4$ by siRNA also induces the downregulation of myofibroblastic markers, such as TGF- $\beta, \alpha$-SMA, collagen $1 \alpha 1$, and vimentin, and the upregulation of the inactivation markers of HSCs, PPAR $\gamma$ and GFAP, with increased numbers of cytoplasmic lipid droplets in LX-2 cells and primary human HSCs [12]. Thus, these results suggest that activated HSCs express T $\beta 4$ in chronically damaged livers, and that this endogenous expression of T $\beta 4$ regulates HSC activation. To support the data for a higher level of T $\beta 4$ expression in the chronically damaged liver, the researchers examined prolyl oligopeptidase (POP) activity in the livers of mice. POP degrades T $\beta 4$ into $N$-acetyl-serylaspartyl-lysyl-proline (Ac-SDKP), which exhibits antifibrotic actions. Ac-SDKP is further degraded into an inactive metabolite by angiotensin-converting enzyme [64]. Kim et al. showed that POP activity decreased in $\mathrm{CCl}_{4}$-treated mice, suggesting that this decrease in POP contributed to the accumulation of T $\beta 4$ in the damaged liver. Chen et al. [65] also supported the notion that preservation of basal Ac-SDKP attenuates $\mathrm{CCl}_{4}$-induced fibrosis in the rat liver; in their research, T $\beta 4$ RNA level was unchanged and POP activity was alleviated, in the livers of rats treated with $\mathrm{CCl}_{4}$ for six weeks. The reduction in POP activity resulted in lower production of Ac-SDKP and higher expression of T $\beta 4$ in livers with chronic injury. In addition, the treatment of T $\beta 4$ with a POP inhibitor significantly increased renal fibrosis in the mice [10]. However, Kim et al.'s experimental system did not investigate the effect of exogenous T $\beta 4$ or the mechanism regarding how T $\beta 4$ regulates the activation of HSCs. Thus, there is no clear explanation for the disparity in the results of Xiao's and Kim's research. Further studies using transgenic mice overexpressing T $\beta 4$ might be helpful for obtaining answers to those questions.

Although the effect of T $\beta 4$ in liver fibrosis remains unclear, it is clear that $\mathrm{T} \beta 4$ is the potential key regulator of HSC activation, and that it can play an essential role in liver disease progression or prevention. Therefore, further studies are required to demonstrate the functions of endogenous T $\beta 4$ in the liver for its future therapeutic application. 


\subsection{Possible Signaling Pathways of Tß4 in Liver Fibrosis}

The PI3K/AKT signaling pathway has been suggested as one of possible targets of T $\beta 4$ in regulating the proliferation and migration of HSCs [11]. In this research, the suppression of T $\beta 4$ induces HSC proliferation and migration by activating the PI3K/AKT pathway. In the cardiac repair process, T $\beta 4$ promotes the migration and survival of cardiac cells by activating ILK [21]. T $\beta 4$ also triggers an EMT in colorectal carcinoma by upregulating ILK [27]. The activity of ILK in these processes is regulated in a PI3K-dependent manner [66,67]. Activated PI3K stimulates ILK activity, which stimulates the phosphorylation of two downstream targets, AKT and GSK-3 $\beta$. Although the effects brought by the T $\beta 4$-PI3K/AKT pathway are varied depending on the cell types, it seems to be associated with cell response to $\mathrm{T} \beta 4$ stimulation.

In renal fibrosis, $\mathrm{T} \beta 4$ is upregulated in glomerulosclerosis and is required for the angiotensin-II-induced expression of PAI-1 (plasminogen activator inhibitor-1) [10]. PAI-1, a major inhibitor of both tissue-type plasminogen activator and urokinase-type plasminogen activator, is a key regulator of fibrinolysis by plasmin. Plasmin directly or indirectly degrades ECM components through the activation of MMPs [68]. Thus, PAI-1 influences organ fibrogenesis by impairing the plasminogen activator. Recent studies have shown that liver damage and fibrosis are ameliorated in PAI-1-deficient mice with BDL [69]. However, in PAI-1-knockout mice after chronic $\mathrm{CCl}_{4}$ exposure, these injuries are dramatically enhanced [70]. Although these studies reported different results depending on the experimental animal model used, the relationship between T $\beta 4$ and PAI-1 might provide clues for understanding the mechanism of T $\beta 4$ in the liver.

\section{Conclusions}

Liver fibrosis is the main characteristic of most chronic liver diseases, and HSCs are known to be the major source of fibrous matrix production. Therefore, the study of the effective regulator that influences the activation of HSCs is considered to provide important clues for developing therapeutics to inhibit liver fibrosis. As discussed above, increased levels of T $\beta 4$ are associated with chronic liver disease and are involved in liver fibrosis by regulating the proliferation and activation of HSCs. Although it has not been established whether T $\beta 4$ promotes or inhibits the activation of HSCs, it is obvious that $\mathrm{T} \beta 4$ is involved in the pathogenesis of liver fibrosis. The involvement of $\mathrm{T} \beta 4$ in fibrogenesis has been reported in several organs and its effect seems to be dependent on the cellular and patho-physiological conditions, implying that the endogenous expression of T $\beta 4$ may be related to disease progression or prevention. The exogenous $\mathrm{T} \beta 4$ peptide is reported to improve cardiac function by rescuing the myocardium in patients with acute myocardial infarction [71], promoting the wound healing process in venous ulcer patients [72], and exerts anti-fibrotic effect in mice. However, the investigation of the therapeutic potential of $\mathrm{T} \beta 4$ should be preceded by an understanding of the role of endogenous $\mathrm{T} \beta 4$ and its interactions with other partners to develop safe and effective treatment for liver disease. Therefore, understanding the potential effects and regulatory mechanism of T $\beta 4$ in liver fibrosis might help to provide a novel treatment for patients with liver fibrosis. 


\section{Acknowledgments}

This work was supported by the National Research Foundation of Korea (NRF) grant funded by the Korea government (MSIP) awarded to Youngmi Jung (2013R1A2A2A01068268, 2014M2B2A9030333).

\section{Author Contributions}

All of the authors wrote the paper.

\section{Conflict of Interests}

The authors declare no conflict of interest.

\section{References}

1. Fattovich, G.; Giustina, G.; Degos, F.; Tremolada, F.; Diodati, G.; Almasio, P.; Nevens, F.; Solinas, A.; Mura, D.; Brouwer, J. Morbidity and mortality in compensated cirrhosis type C: A retrospective follow-up study of 384 patients. Gastroenterology 1997, 112, 463-472.

2. Bica, I.; McGovern, B.; Dhar, R.; Stone, D.; McGowan, K.; Scheib, R.; Snydman, D.R. Increasing mortality due to end-stage liver disease in patients with human immunodeficiency virus infection. Clin. Infect. Dis. 2001, 32, 492-497.

3. Wong, J.B.; McQuillan, G.M.; McHutchison, J.G.; Poynard, T. Estimating future hepatitis C morbidity, mortality and costs in the united states. Am. J. Public Health 2000, 90, 1562.

4. Yuen, M.F.; Hou, J.L.; Chutaputti, A. Hepatocellular carcinoma in the asia pacific region. J. Gastroenterol. Hepatol. 2009, 24, 346-353.

5. Pinzani, M.; Rombouts, K. Liver fibrosis: From the bench to clinical targets. Digest. Liver Dis. 2004, 36, 231-242.

6. Schuppan, D.; Afdhal, N.H. Liver cirrhosis. The Lancet 2008, 371, 838-851.

7. Bataller, R.; Brenner, D.A. Liver fibrosis. J. Clin. Investig. 2005, 115, 209.

8. Friedman, S.L. Hepatic stellate cells: Protean, multifunctional, and enigmatic cells of the liver. Physiol. Rev. 2008, 88, 125-172.

9. Goldstein, A.L.; Hannappel, E.; Kleinman, H.K. Thymosin $\beta 4$ : Actin-sequestering protein moonlights to repair injured tissues. Trends Mol. Med. 2005, 11, 421-429.

10. Zuo, Y.; Chun, B.; Potthoff, S.A.; Kazi, N.; Brolin, T.J.; Orhan, D.; Yang, H.-C.; Ma, L.-J.; Kon, V.; Myöhänen, T. Thymosin $\beta 4$ and its degradation product, ac-sdkp, are novel reparative factors in renal fibrosis. Kidney Int. 2013, 84, 1166-1175.

11. Xiao, Y.; Qu, C.; Ge, W.; Wang, B.; Wu, J.; Xu, L.; Chen, Y. Depletion of thymosin $\beta 4$ promotes the proliferation, migration, and activation of human hepatic stellate cells. Cell. Physiol. Biochem. 2014, 34, 356-367.

12. Kim, J.; Wang, S.; Hyun, J.; Choi, S.S.; Cha, H.; Ock, M.; Jung, Y. Hepatic stellate cells express thymosin beta 4 in chronically damaged liver. PLOS ONE 2015, 10, e0122758.

13. Goldstein, A.L.; Slater, F.D.; White, A. Preparation, assay, and partial purification of a thymic lymphocytopoietic factor (thymosin). Proc. Natl. Acad. Sci. USA 1966, 56, 1010. 
14. Low, T.; Hu, S.-K.; Goldstein, A.L. Complete amino acid sequence of bovine thymosin beta 4: A thymic hormone that induces terminal deoxynucleotidyl transferase activity in thymocyte populations. Proc. Natl. Acad. Sci. USA 1981, 78, 1162-1166.

15. Sanders, M.C.; Goldstein, A.L.; Wang, Y.-L. Thymosin beta 4 (fx peptide) is a potent regulator of actin polymerization in living cells. Proc. Natl. Acad. Sci. USA 1992, 89, 4678-4682.

16. Cha, H.-J.; Jeong, M.-J.; Kleinman, H.K. Role of thymosin $\beta 4$ in tumor metastasis and angiogenesis. J. Natl. Cancer Inst. 2003, 95, 1674-1680.

17. Philp, D.; Goldstein, A.; Kleinman, H. Thymosin $\beta 4$ promotes angiogenesis, wound healing, and hair follicle development. Mech. Ageing Dev. 2004, 125, 113-115.

18. Philp, D.; St-surin, S.; Cha, H.J.; Moon, H.S.; Kleinman, H.K.; Elkin, M. Thymosin beta 4 induces hair growth via stem cell migration and differentiation. Ann. N. Y. Acad. Sci. 2007, 1112, 95-103.

19. Sosne, G.; Szliter, E.A.; Barrett, R.; Kernacki, K.A.; Kleinman, H.; Hazlett, L.D. Thymosin beta 4 promotes corneal wound healing and decreases inflammation in vivo following alkali injury. Exp. Eye Res. 2002, 74, 293-299.

20. Malinda, K.M.; Sidhu, G.S.; Mani, H.; Banaudha, K.; Maheshwari, R.K.; Goldstein, A.L.; Kleinman, H.K. Thymosin $\beta 4$ accelerates wound healing. J. Investig. Dermatol. 1999, 113, 364-368.

21. Bock-Marquette, I.; Saxena, A.; White, M.D.; DiMaio, J.M.; Srivastava, D. Thymosin $\beta 4$ activates integrin-linked kinase and promotes cardiac cell migration, survival and cardiac repair. Nature 2004, 432, 466-472.

22. Larsson, L.-I.; Holck, S. Occurrence of thymosin $\beta 4$ in human breast cancer cells and in other cell types of the tumor microenvironment. Hum. Pathol. 2007, 38, 114-119.

23. Ji, Y.-I.; Lee, B.-Y.; Kang, Y.-J.; Jo, J.-O.; Lee, S.H.; Kim, H.Y.; Kim, Y.-O.; Lee, C.; Koh, S.B.; Kim, A. Expression patterns of thymosin $\beta 4$ and cancer stem cell marker cd133 in ovarian cancers. Pathol. Oncol. Res. 2013, 19, 237-245.

24. Goldstein, A.L. Thymosin $\beta 4$ : A new molecular target for antitumor strategies. J. Natl. Cancer Inst. 2003, 95, 1646-1647.

25. Wang, W.-S.; Chen, P.-M.; Hsiao, H.-L.; Wang, H.-S.; Liang, W.-Y.; Su, Y. Overexpression of the thymosin $\beta-4$ gene is associated with increased invasion of sw480 colon carcinoma cells and the distant metastasis of human colorectal carcinoma. Oncogene 2004, 23, 6666-6671.

26. Kobayashi, T.; Okada, F.; Fujii, N.; Tomita, N.; Ito, S.; Tazawa, H.; Aoyama, T.; Choi, S.K.; Shibata, T.; Fujita, H. Thymosin- $\beta 4$ regulates motility and metastasis of malignant mouse fibrosarcoma cells. Am. J. Pathol. 2002, 160, 869-882.

27. Huang, H.; Hu, C.; Tang, M.; Wang, W.; Chen, P.; Su, Y. Thymosin $\beta 4$ triggers an epithelial-mesenchymal transition in colorectal carcinoma by upregulating integrin-linked kinase. Oncogene 2007, 26, 2781-2790.

28. Moon, E.-Y.; Im, Y.-S.; Ryu, Y.-K.; Kang, J.-H. Actin-sequestering protein, thymosin beta-4, is a novel hypoxia responsive regulator. Clin. Exp. Metastasis 2010, 27, 601-609.

29. Sosne, G.; Xu, L.; Prach, L.; Mrock, L.K.; Kleinman, H.K.; Letterio, J.J.; Hazlett, L.D.; Kurpakus-Wheater, M. Thymosin beta 4 stimulates laminin-5 production independent of TGF-beta. Exp. Cell Res. 2004, 293, 175-183. 
30. Ryu, Y.K.; Lee, Y.S.; Lee, G.H.; Song, K.S.; Kim, Y.S.; Moon, E.Y. Regulation of glycogen synthase kinase-3 by thymosin beta-4 is associated with gastric cancer cell migration. Int. J. Cancer 2012, 131, 2067-2077.

31. Oh, I.S.; So, S.S.; Jahng, K.Y.; Kim, H.G. Hepatocyte growth factor upregulates thymosin $\beta 4$ in human umbilical vein endothelial cells. Biochem. Biophys. Res. Commun. 2002, 296, 401-405.

32. Reeves, H.L.; Friedman, S.L. Activation of hepatic stellate cells-a key issue in liver fibrosis. Front. Biosci. 2002, 7, 808-826.

33. Friedman, S.L. Mechanisms of hepatic fibrogenesis. Gastroenterology 2008, 134, 1655-1669.

34. Gard, A.L.; White, F.P.; Dutton, G.R. Extra-neural glial fibrillary acidic protein (gfap) immunoreactivity in perisinusoidal stellate cells of rat liver. J. Neuroimmunol. 1985, 8, 359-375.

35. Friedman, S.L. Molecular regulation of hepatic fibrosis, an integrated cellular response to tissue injury. J. Biol. Chem. 2000, 275, 2247-2250.

36. Li, D.; Friedman, S. Liver fibrogenesis and the role of hepatic stellate cells: New insights and prospects for therapy. J. Gastroenterol. Hepatol. 1999, 14, 618-633.

37. Bataller, R.; Brenner, D.A. Hepatic stellate cells as a target for the treatment of liver fibrosis. Semin. Liver Dis. 2001, 21, 437-451.

38. Pinzani, M. Pdgf and signal transduction in hepatic stellate cells. Front. Biosci. 2002, 7, d1720-d1726.

39. Wang, Y.; Jiang, X.-Y.; Liu, L.; Jiang, H.-Q. Phosphatidylinositol 3-kinase/akt pathway regulates hepatic stellate cell apoptosis. World J. Gastroenterol. 2008, 14, 5186.

40. Omenetti, A.; Choi, S.; Michelotti, G.; Diehl, A.M. Hedgehog signaling in the liver. J. Hepatol. 2011, 54, 366-373.

41. Gressner, A.M.; Weiskirchen, R.; Breitkopf, K.; Dooley, S. Roles of tgf-beta in hepatic fibrosis. Front. Biosci. 2002, 7, d793-d807.

42. Reif, S.; Lang, A.; Lindquist, J.N.; Yata, Y.; Gäbele, E.; Scanga, A.; Brenner, D.A.; Rippe, R.A. The role of focal adhesion kinase-phosphatidylinositol 3-kinase-akt signaling in hepatic stellate cell proliferation and type i collagen expression. J. Biol. Chem. 2003, 278, 8083-8090.

43. Sicklick, J.K.; Li, Y.-X.; Choi, S.S.; Qi, Y.; Chen, W.; Bustamante, M.; Huang, J.; Zdanowicz, M.; Camp, T.; Torbenson, M.S. Role for hedgehog signaling in hepatic stellate cell activation and viability. Lab. Investig. 2005, 85, 1368-1380.

44. Choi, S.S.; Omenetti, A.; Witek, R.P.; Moylan, C.A.; Syn, W.-K.; Jung, Y.; Yang, L.; Sudan, D.L.; Sicklick, J.K.; Michelotti, G.A. Hedgehog pathway activation and epithelial-to-mesenchymal transitions during myofibroblastic transformation of rat hepatic cells in culture and cirrhosis. Am. J. Physiol.-Gastrointest. Liver Physiol. 2009, 297, G1093-G1106.

45. Bi, W.-R.; Yang, C.-Q.; Shi, Q. Transforming growth factor- $\beta 1$ induced epithelial-mesenchymal transition in hepatic fibrosis. Hepato-Gastroenterol. 2012, 59, 1960-1963.

46. Nemolato, S.; van Eyken, P.; Cabras, T.; Cau, F.; Fanari, M.; Locci, A.; Fanni, D.; Gerosa, C.; Messana, I.; Castagnola, M. Expression pattern of thymosin beta 4 in the adult human liver. Eur. J. Histochem. 2011, 55, e25.

47. Paulussen, M.; Landuyt, B.; Schoofs, L.; Luyten, W.; Arckens, L. Thymosin beta 4 mrna and peptide expression in phagocytic cells of different mouse tissues. Peptides 2009, 30, 1822-1832. 
48. Theunissen, W.; Fanni, D.; Nemolato, S.; di Felice, E.; Cabras, T.; Gerosa, C.; van Eyken, P.; Messana, I.; Castagnola, M.; Faa, G. Thymosin beta 4 and thymosin beta 10 expression in hepatocellular carcinoma. Eur. J. Histochem. 2014, 58, 2242.

49. Fu, X.; Cui, P.; Chen, F.; Xu, J.; Gong, L.; Jiang, L.; Zhang, D.; Xiao, Y. Thymosin $\beta 4$ promotes hepatoblastoma metastasis via the induction of epithelial-mesenchymal transition. Mol. Med. Rep. 2015, 12, 127-132.

50. Barnaeva, E.; Nadezhda, A.; Hannappel, E.; Sjogren, M.H.; Rojkind, M. Thymosin $\beta 4$ upregulates the expression of hepatocyte growth factor and downregulates the expression of pdgf- $\beta$ receptor in human hepatic stellate cells. Ann. N. Y. Acad. Sci. 2007, 1112, 154-160.

51. Schirmacher, P.; Geerts, A.; Pietrangelo, A.; Dienes, H.P.; Rogler, C.E. Hepatocyte growth factor/hepatopoietin a is expressed in fat-storing cells from rat liver but not myofibroblast - like cells derived from fat - storing cells. Hepatology 1992, 15, 5-11.

52. Pinzani, M.; Gentilini, A.; Caligiuri, A.; de Franco, R.; Pellegrini, G.; Milani, S.; Marra, F.; Gentilini, P. Transforming growth factor- $\beta 1$ regulates platelet - derived growth factor receptor $\beta$ subunit in human liver fat - storing cells. Hepatology 1995, 21, 232-239.

53. Rockey, D.C.; Boyles, J.; Gabbiani, G.; Friedman, S. Rat hepatic lipocytes express smooth muscle actin upon activation in vivo and in culture. J. Submicrosc. Cytol. Pathol. 1992, 24, 193-203.

54. Jiang, F.; Parsons, C.J.; Stefanovic, B. Gene expression profile of quiescent and activated rat hepatic stellate cells implicates wnt signaling pathway in activation. J. Hepatol. 2006, 45, 401-409.

55. Cheng, J.H.; She, H.; Han, Y.-P.; Wang, J.; Xiong, S.; Asahina, K.; Tsukamoto, H. Wnt antagonism inhibits hepatic stellate cell activation and liver fibrosis. Am. J. Physiol.-Gastrointest. Liver Physiol. 2008, 294, G39-G49.

56. Reyes-Gordillo, K.; Shah, R.; Popratiloff, A.; Fu, S.; Hindle, A.; Brody, F.; Rojkind, M. Thymosin- $\beta 4(\mathrm{t} \beta 4)$ blunts pdgf-dependent phosphorylation and binding of akt to actin in hepatic stellate cells. Am. J. Pathol. 2011, 178, 2100-2108.

57. Reyes-Gordillo, K.; Shah, R.; Arellanes - Robledo, J.; Rojkind, M.; Lakshman, M.R. Protective effects of thymosin $\beta 4$ on carbon tetrachloride - induced acute hepatotoxicity in rats. Ann. N. Y. Acad. Sci. 2012, 1269, 61-68.

58. Mann, J.; Oakley, F.; Akiboye, F.; Elsharkawy, A.; Thorne, A.W.; Mann, D.A. Regulation of myofibroblast transdifferentiation by DNA methylation and mecp2: Implications for wound healing and fibrogenesis. Cell Death Differ. 2007, 14, 275-285.

59. Henkel, C.; Schwamborn, K.; Zimmermann, H.W.; Tacke, F.; Kühnen, E.; Odenthal, M.; Groseclose, M.R.; Caprioli, R.M.; Weiskirchen, R. From proteomic multimarker profiling to interesting proteins: Thymosin- $\beta 4$ and kininogen-1 as new potential biomarkers for inflammatory hepatic lesions. J. Cell. Mol. Med. 2011, 15, 2176-2188.

60. Kountouras, J.; Billing, B.H.; Scheuer, P.J. Prolonged bile duct obstruction: A new experimental model for cirrhosis in the rat. Br. J. Exp. Pathol. 1984, 65, 305.

61. Luk, J.M.; Zhang, Q.S.; Lee, N.P.; Wo, J.Y.; Leung, P.P.; Liu, L.X.; Hu, M.Y.; Cheung, K.F.; Hui, C.K.; Lau, G.K.; et al. Hepatic stellate cell-targeted delivery of m6p-has-glycyrrhetinic acid attenuates hepatic fibrogenesis in a bile duct ligation rat model. Liver Int. 2007, 27, 548-557. 
62. Giebeler, A.; Boekschoten, M.V.; Klein, C.; Borowiak, M.; Birchmeier, C.; Gassler, N.; Wasmuth, H.E.; Müller, M.; Trautwein, C.; Streetz, K.L. C-met confers protection against chronic liver tissue damage and fibrosis progression after bile duct ligation in mice. Gastroenterology 2009, 137, 297-308.

63. Chen, S.; Chen, Y.; Zhang, X.; Qian, H.; Chen, W.; Xie, W. Targeted inhibition of platelet-derived growth factor receptor- $\beta$ subunit in hepatic stellate cells ameliorates hepatic fibrosis in rats. Gene Ther. 2008, 15, 1424-1435.

64. Cavasin, M.A.; Liao, T.-D.; Yang, X.-P.; Yang, J.J.; Carretero, O.A. Decreased endogenous levels of ac-sdkp promote organ fibrosis. Hypertension 2007, 50, 130-136.

65. Chen, Y.-W.; Liu, B.-W.; Zhang, Y.-J.; Chen, Y.-W.; Dong, G.-F.; Ding, X.-D.; Xu, L.-M.; Pat, B.; Fan, J.-G.; Li, D.-G. Preservation of basal acsdkp attenuates carbon tetrachloride-induced fibrosis in the rat liver. J. Hepatol. 2010, 53, 528-536.

66. Legate, K.R.; Montañez, E.; Kudlacek, O.; Füssler, R. Ilk, pinch and parvin: The tipp of integrin signalling. Nat. Rev. Mol. Cell Biol. 2006, 7, 20-31.

67. McDonald, P.C.; Fielding, A.B.; Dedhar, S. Integrin-linked kinase-essential roles in physiology and cancer biology. J. Cell Sci. 2008, 121, 3121-3132.

68. Ghosh, A.K.; Vaughan, D.E. Pai-1 in tissue fibrosis. J. Cell. Physiol. 2012, 227, 493-507.

69. Bergheim, I.; Guo, L.; Davis, M.A.; Duveau, I.; Arteel, G.E. Critical role of plasminogen activator inhibitor-1 in cholestatic liver injury and fibrosis. J. Pharmacol. Exp. Ther. 2006, 316, 592-600.

70. Von Montfort, C.; Beier, J.I.; Kaiser, J.P.; Guo, L.; Joshi-Barve, S.; Pritchard, M.T.; Arteel, G.E. Pai-1 plays a protective role in ccl4-induced hepatic fibrosis in mice: Role of hepatocyte division. Am. J. Physiol.-Gastrointest. Liver Physiol. 2010, 298, G657-G666.

71. Crockford, D. Development of thymosin $\beta 4$ for treatment of patients with ischemic heart disease. Ann. N. Y. Acad. Sci. 2007, 1112, 385-395.

72. Guarnera, G.; de Rosa, A.; Camerini, R. Thymosin $\beta-4$ and venous ulcers. Ann. N. Y. Acad. Sci. 2007, 1112, 407-412.

(C) 2015 by the authors; licensee MDPI, Basel, Switzerland. This article is an open access article distributed under the terms and conditions of the Creative Commons Attribution license (http://creativecommons.org/licenses/by/4.0/). 